\title{
Application of continuous wavelet transform to the analysis of the modulus of the fractional Fourier transform bands for resolving two componentmixture
}

\author{
Erdal Dinç • Fernando B. Duarte •
}

J. A. Tenreiro Machado · Dumitru Baleanu

\begin{abstract}
In this paper, the fractional Fourier transform (FrFT) is applied to the spectral bands of two component mix- ture containing oxfendazole and oxyclozanide to provide the multicomponent quantitative prediction of the related sub- stances. With this aim in mind, the modulus of FrFT spectral bands are processed by the continuous Mexican Hat family of wavelets, being denoted by MEXH-CWTMOFrFT. Four modulus sets are obtained for the parameter $a$ of the FrFT going from 0.6 up to 0.9 in order to compare their effects upon the spectral and quantitative resolutions. Four linear regres- sion plots for each substance were obtained by measuring the MEXH-CWT-MOFrFT amplitudes in the application of the MEXH family to the modulus of the FrFT. This new com- bined powerful tool is validated by analyzing the artificial samples of the related drugs, and it is applied to the quality control of the commercial veterinary samples.
\end{abstract}

Keywords Fractional Fourier transform · Continuous wavelet transform · Spectral resolution · Quantitative analysis · Binary mixture

\section{Introduction}

In recent years, the rapid developments of the signal process- ing tools and applications in different fields were used to provide many opportunities for resolving the complex spec- tral bands for qualitative and quantitative evaluations. Within this context, the first problem is the overlapping spectra of the analyzed drugs in the same wavelength region. Second prob- lem is the sensitivity due to the ratio of signal to noise. In [1], the main purpose was to evaluate the effects of anthelmintic treatment with oxfendazole (OXF) and oxyclozanide (OXY) in different formulation (paste and tablet) on parasite load and metabolic status in sheep. It was proved that a com- bination of OXF and $O X Y$ becomes effective to eliminate parasite burden in sheep. In [2], the bivariate calibration and the multivariate spectral calibration were applied for the simultaneous determination of OXF and OXY in their syn- thetic binary mixtures and commercial veterinary formula- tion without requiring a priory separation step. Continuous wavelet transform (CWT) was applied to the absorption sig- nals and their ratio signals for the analysis of mixture of OXF and OXY in bolus. The method performances of CWT- zero crossing and ratio spectra-CWT techniques were vali- dated by using the synthetic mixtures and by applying the 
standard addition technique, and successfully, accuracy, pre- cision and reproducibility results were reported [3]. Chemo- metric multivariate calibration methods were applied to the spectrophotometric analysis of a veterinary formulation containing OXF and OXY compounds [4]. The simultaneous determination of OXF and OXY in a pharmaceutical prepara- tion was performed by reversed phase chromatography with isocratic elution. The validity of the method was evaluated in terms of linear regression analysis, precision, specificity and accuracy [5]. Three new spectrophotometric methods are described for the determination of OXF and $\mathrm{OXY}$ in their binary mixture: first derivative spectrophotometry, ratio spectra derivative and Vierordt's method [6].

In this study, a new approach based on the application of the CWT to the fractional Fourier transform (FrFT) coeffi- cients is proposed for the multicomponent determination of OXF and OXY in commercial veterinary samples without using any chemical pretreatments.

The paper is organized as follows. Section 2 introduces the fundamentals of the FrFT. Section 3 presents the 4 experimental setup. Section 5 discusses the results. Finally, outlines the main conclusions.

\section{The fractional Fourier transform method}

The FrFT is a generalization of the ordinary Fourier trans- form with an order parameter $a \in \mathrm{R}$. Mathematically, the ath order fractional Fourier transform (FT) $\mathrm{FrFT}^{a}$ is the $a$ th power of the ordinary FT operation.

With the development of the FrFT and related concepts, we see that the ordinary frequency domain is merely a special case of a continuum of fractional Fourier domains, which are intimately related to time-frequency (or space-frequency) representations.

Every property and application of the ordinary FT becomes a special case of the FrFT. In all areas where FTs and frequencydomain concepts are used, there exists the potential for generalization and improvement by using the FrFT.

The FrFT has been found to play an important role in the study of optical systems known as Fourier optics, with appli- cations in optical information processing, allowing a refor-mulation of this area in a much more general way. It has also generalized the notion of the frequency domain and extended our understanding of the time-frequency plane, two central concepts in signal analysis and signal processing. FrFT is expected to have an impact in the form of deeper understand- ing or new applications in every area in which the FT plays a significant role, and to take its place among the standard mathematical tools of physics and engineering.

FrFT in the form of fractional powers of the Fourier oper- ator appears in the mathematical literature as early as 1929
[7]. Later on it was used in quantum mechanics and signal processing $[8,9]$, but it was mainly the optical interpretation and the applications in optics that gave a burst of publications since the nineties that culminated in the book of Ozaktas et al. [10]. In this book are presented several definitions of the FrFT. All of them were suggested to be used in different con- texts like the voice, images or signal processing and work well with the fractional models, but there is no known direct connection between these definitions and the Fractional Cal- culus (FC). The answer to the question what definition to use depends mainly on the problem we are dealing with. There is not a 'best' definition of the FrFT, and one should rather try to take the most suitable one while modeling a process or considering a mathematical problem [1113].

The FT of a function can be considered as a linear differential operator acting on that function, while the FrFT generalizes this differential operator by letting it depend on a continuous parameter a. In mathematical terms, the ath order FrFT is the ath power of FT operator.

Several FrFT definitions are found in the literature, which converge to the original definition. Among them, the most commonly used the ath order fractional Fourier transform of a function $s(t)$ is a linear operation defined by:

$$
F r F T^{\alpha}\{s(u)\}=\int_{-\infty}^{+\infty} k_{\alpha}(u, t) s(t) \mathrm{d} t
$$

where $\alpha$ indicates the rotation angle in the time-frequency plane, $u$ is the frequency domain, $k_{\alpha}(u, t) s(t)$ is the kernel function shown acknowledgments in the equation:

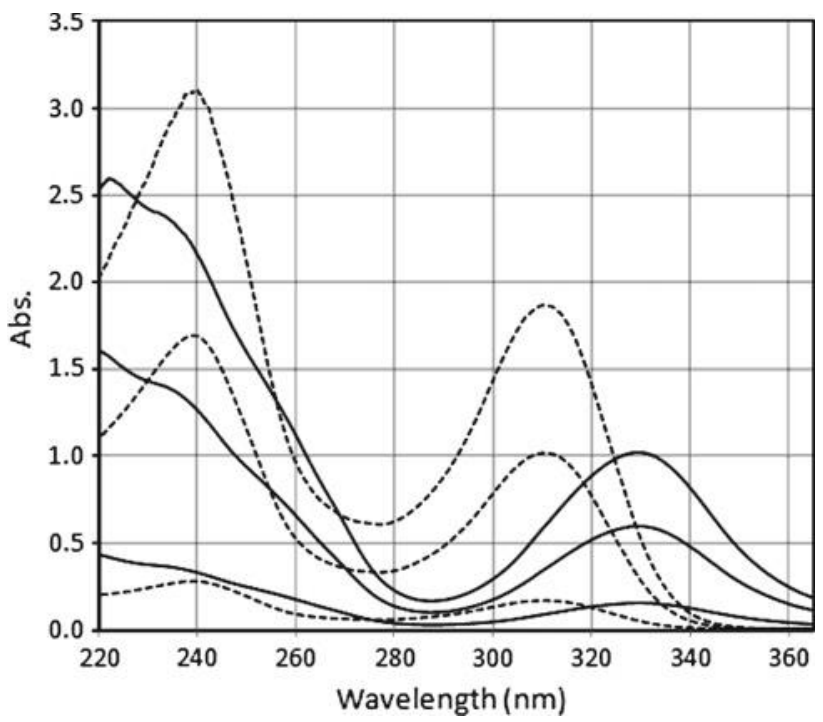

Fig. 1 Spectral bands of OXF (continuous line) and OXY (dashed line) in the range between $2-26$ and $4-28 \mu \mathrm{g} / \mathrm{mL}$ 

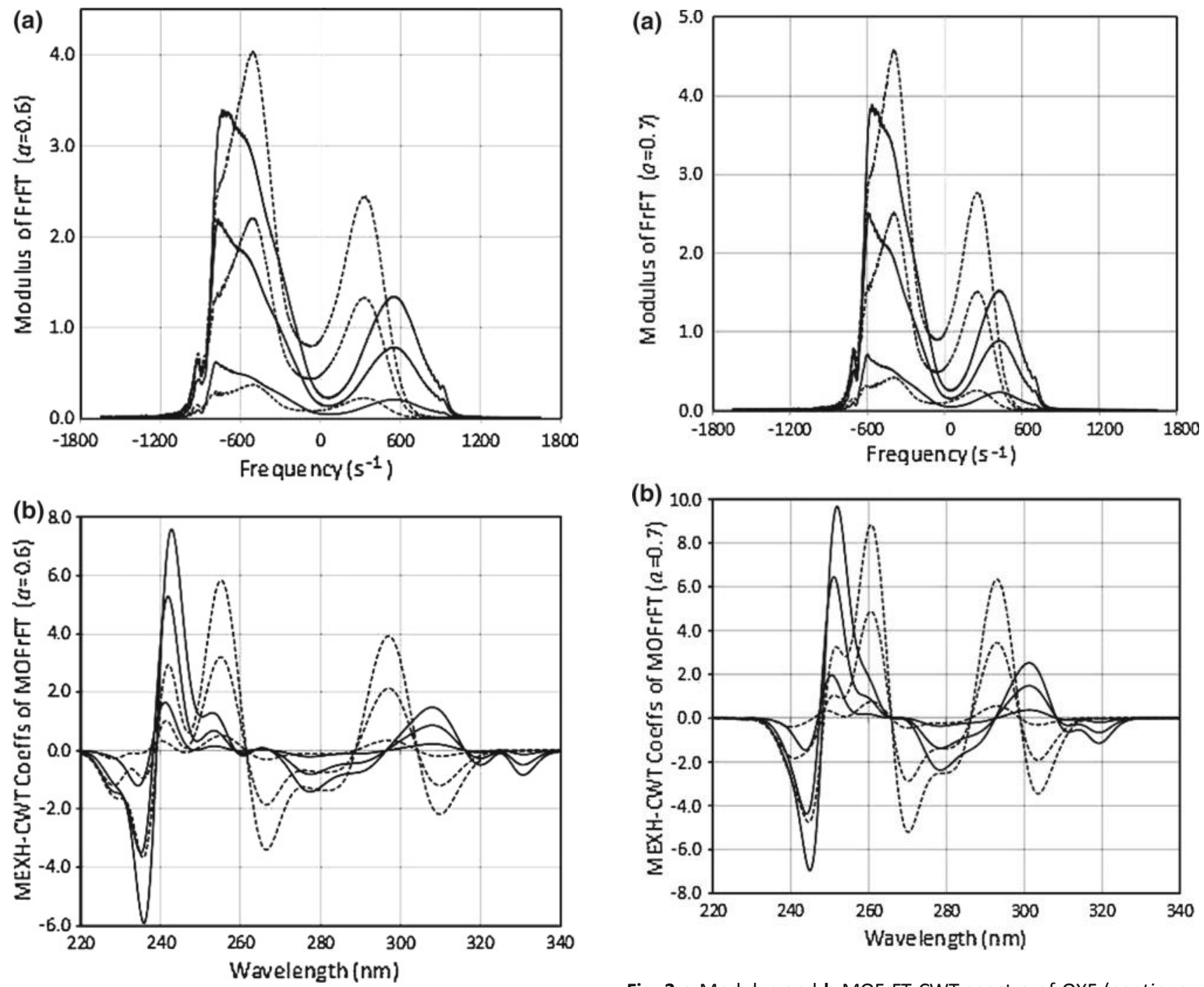

Fig. 2 a Modulus and b MOFrFT-CWT spectra corresponding to OXF (continuous line) and OXY (dashed line) in the range between $2-26$ and $4-28 \mu \mathrm{g} / \mathrm{mL}(a=0.6)$

$$
k_{\alpha}(u, t)= \begin{cases}\sqrt{\frac{1-i \cot (\alpha)}{2 \pi}} e^{i\left[\frac{t^{2}+u^{2}}{2} \cot (\alpha)-\csc (\alpha) u t\right]}, & \alpha \neq n \pi \\ \delta(t-u) & \alpha=2 n \pi \\ \delta(t+u) & \alpha=2 n \pi \pm \pi\end{cases}
$$

where $n \in$ Zand $i=\stackrel{V}{ }=-1[14]$.

FrThe aten order transform is sometimes referred to as $\alpha$ th order transform, a practice which will occasionally be found convenient when no confusion can arise. The FrFT has the following special cases:

$$
\begin{aligned}
& \operatorname{FrFT}^{2 n}\{s(u)\}=s(u) \\
& \operatorname{FrFT}^{2 n \pi+\frac{\pi}{2}}\{s(u)\}=F T\{s(u)\}
\end{aligned}
$$

Fig. 3 a Modulus and $\mathbf{b}$ MOFrFT-CWT spectra of OXF (continuous line) and OXY (dashed line) in the range between 2-26 and 4-28 $\mu \mathrm{g} / \mathrm{mL}(a=0.7)$

The time domain is the FrFT domain with $\alpha=2 n \pi$, while the frequency domain is the FrFT domain with $\alpha=2 n \pi+2$.

Since the FrFT is periodic, with period $2^{\pi, \alpha}$ can be limited in the interval $[-\pi, \pi][9]$.

Several algorithms for the discrete implementation of the FrFT were proposed $[9,14,15]$. Nevertheless, since the cal-

$$
\begin{aligned}
& \operatorname{FrFT}^{2 n \pi \pm \pi}\{s(u)\}=s(-u) \\
& \operatorname{FrFT}^{2 n \pi-\frac{\pi}{2}}\{s(u)\}=F T\{s(-u)\}
\end{aligned}
$$


culation speed is not the main issue in the study, the authors decided to program directly the continuous version defined by Eqs. (3)-(6).

\section{Experimental setup}

A Shimadzu UV-160 double beam UV-VIS spectrophotome- ter with a fixed slit width $(2 \mathrm{~nm})$ connected to a com- puter equipped with Shimadzu UVPC software, and an HP LaserJet P1505 printer were used for the registration 

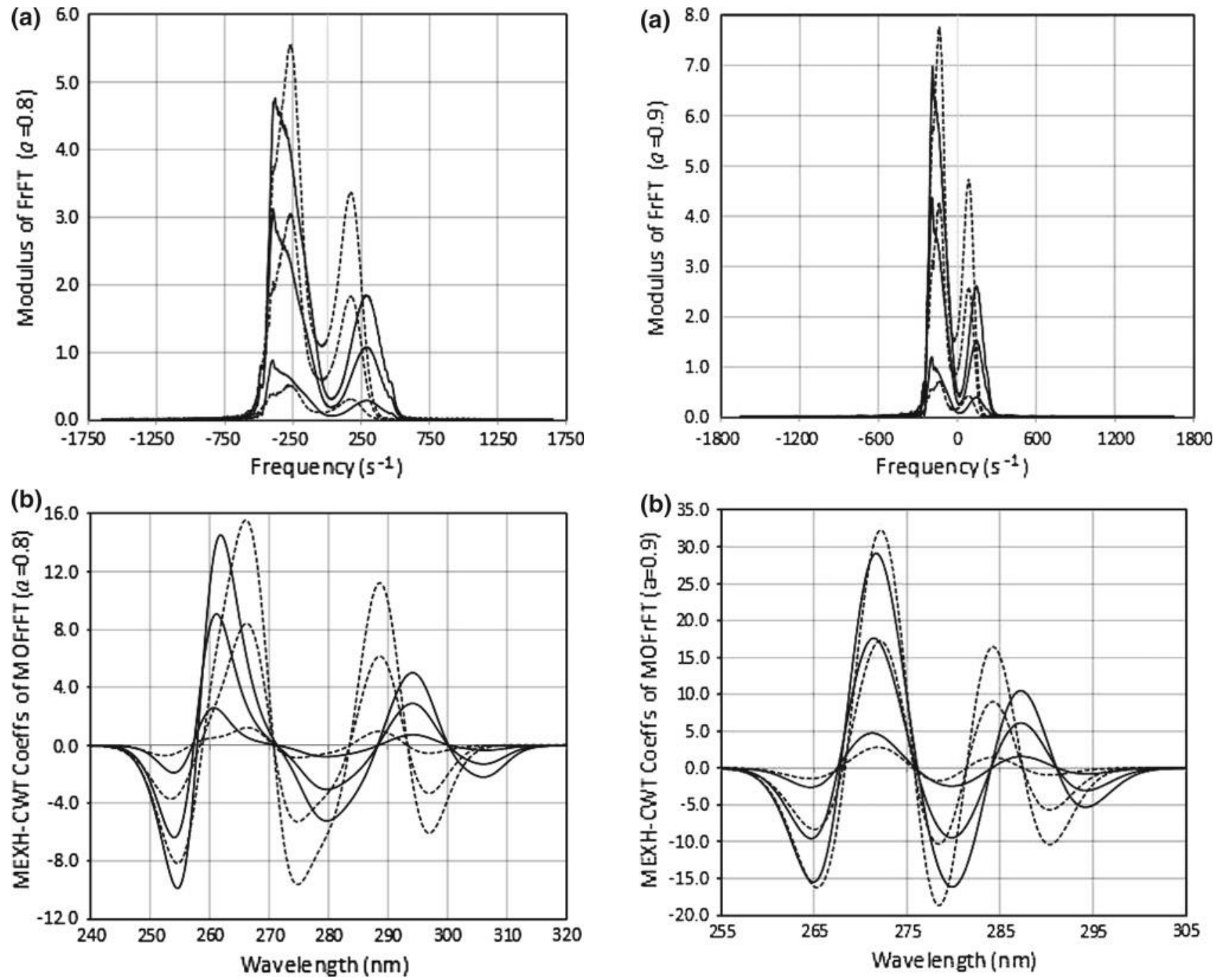

Fig. 4 a Modulus and b MOFrFT-CWT spectra corresponding to OXF (continuous line) and OXY (dashed line) in the range between $2-26$ and $4-28 \mu \mathrm{g} / \mathrm{mL}(a=0.8)$

of the absorption spectra. All data treatments, regressions and statistical analysis were obtained within the Matlab software.

Stock solution of $25 \mathrm{mg} / 50 \mathrm{~mL}$ OXY and OXF were pre- pared in $0.1 \mathrm{M} \mathrm{NaOH}$ and methanol $(50: 50, v / v)$. Calibra- tion solutions of OXF and OXY in the concentration range of 2-26 and 4-28 $\mu \mathrm{g} / \mathrm{mL}$ were prepared by using the stock solutions. The artificial mixture solutions were used for the methodvalidation.

The UV-spectra of the calibration and validation samples were plotted by using a Shimadzu UV-2550 spectrophotome- ter. In the sequel, the FrFT is applied to the UV-spectra of the samples of the related compounds. The spectra of FrFT coef- ficients are then processed by using MEXH-CWT method for the multicomponent determination of OXF and OXY in commercial veterinary samples.

Fig. 5 a Modulus and b MOFrFT-CWT spectra of OXF (continuous line) and OXY (dashed line) in the range between 2-26 and 4-28 $\mu \mathrm{g} / \mathrm{mL}(a=0.9)$

\section{Results and discussion}

Figure 1 indicates the absorption spectral bands of the calibration solution of OXF (-) and OXY (-) in the range of 2-26 and 4-28 $\mu \mathrm{g} / \mathrm{mL}$, respectively. The same spectral band registration procedure was applied to the artificial and com- mercial samples of OXF and OXY.

In this work, a new signal processing approach is used for the quantitative resolution of the OXF-OXY mixtures to reach better determination results in the presence of the overlapping spectral band. Having this aim in mind, we apply

the FrFT at four different parameters $a=\{0.6,0.7,0.8,0.9\}$ to obtain the modulus of the UV-spectral bands as shown in Figs. 2a, 3a, $4 a$ and $5 a$, respectively.

The modulus obtained by the FrFT is processed by MEXHCWT tool to provide the resolved spectral bands. 
Table 1 Calibration data obtained by using the least square

\begin{tabular}{|c|c|c|c|c|c|c|c|c|}
\hline \multirow[t]{2}{*}{ Parameter } & \multicolumn{2}{|l|}{$a=0.6$} & \multicolumn{2}{|l|}{$a=0.7$} & \multicolumn{2}{|l|}{$a=0.8$} & \multicolumn{2}{|l|}{$a=0.9$} \\
\hline & OXF & OXY & OXF & OXY & OXF & OXY & OXF & OXY \\
\hline & 296.3 & 288.6 & 292.4 & 286.1 & 288.2 & 283.4 & 284 & 281.3 \\
\hline$m$ & $1.48 \mathrm{E}-01$ & $-2.72 \mathrm{E}-02$ & $2.39 \mathrm{E}-01$ & $-5.15 \mathrm{E}-02$ & $4.24 \mathrm{E}-01$ & $-1.39 \mathrm{E}-01$ & $6.22 \mathrm{E}-01$ & $-4.76 \mathrm{E}-01$ \\
\hline$n$ & $4.37 \mathrm{E}-02$ & $-6.15 \mathrm{E}-03$ & $7.80 \mathrm{E}-02$ & $-1.21 \mathrm{E}-02$ & $1.48 \mathrm{E}-01$ & $-4.13 \mathrm{E}-02$ & $2.05 \mathrm{E}-01$ & $-2.05 \mathrm{E}-01$ \\
\hline$r$ & 1.0000 & 1.0000 & 1.0000 & 1.0000 & 1.0000 & 0.9999 & 1.0000 & 0.9999 \\
\hline$S E(m)$ & $1.01 \mathrm{E}-04$ & $2.28 \mathrm{E}-04$ & $2.42 \mathrm{E}-04$ & $4.81 \mathrm{E}-04$ & $9.43 \mathrm{E}-04$ & $1.53 \mathrm{E}-03$ & $8.45 \mathrm{E}-04$ & $6.61 \mathrm{E}-03$ \\
\hline$S E(n)$ & $3.28 \mathrm{E}-03$ & $3.28 \mathrm{E}-03$ & $6.90 \mathrm{E}-03$ & $6.90 \mathrm{E}-03$ & $1.29 \mathrm{E}-02$ & $1.29 \mathrm{E}-02$ & $5.12 \mathrm{E}-02$ & $5.12 \mathrm{E}-02$ \\
\hline$S E(r)$ & $1.72 \mathrm{E}-03$ & $3.87 \mathrm{E}-03$ & $4.11 \mathrm{E}-03$ & $8.17 \mathrm{E}-03$ & $1.60 \mathrm{E}-02$ & $2.60 \mathrm{E}-02$ & $1.43 \mathrm{E}-02$ & $1.12 \mathrm{E}-01$ \\
\hline LOD & 0.06 & 0.63 & 0.09 & 0.70 & 0.20 & 0.48 & 0.12 & 0.56 \\
\hline LOQ & 0.20 & 2.09 & 0.30 & 2.32 & 0.66 & 1.60 & 0.40 & 1.86 \\
\hline
\end{tabular}

$m$ slope of the linear regression equation, $n$ intercept of the linear regression equation, $r$ correlation coefficient of the linear regression equation, $S E(m)$ standard error of the slope, $S E(n)$ standard error of the intercept, $S E(r)$ standard error of the correlation coefficient, $L O D$ limit of detection, $L O Q$ limit of quantization

Table 2 Validation results of OXY and OXF in the artificial mixture samples by the new proposed

\begin{tabular}{|c|c|c|c|c|c|c|c|c|c|c|}
\hline \multirow[t]{2}{*}{ No. } & \multirow[t]{2}{*}{$\begin{array}{l}\text { Binary mix. } \\
\text { OXY }\end{array}$} & \multirow[t]{2}{*}{$\begin{array}{l}\text { OXF } \\
\text { OXF }\end{array}$} & \multicolumn{2}{|c|}{$\begin{array}{l}a=0.6 \\
\text { Recovery }(\%)\end{array}$} & \multicolumn{2}{|c|}{$\begin{array}{l}a=0.7 \\
\text { Recovery }(\%)\end{array}$} & \multicolumn{2}{|c|}{$\begin{array}{l}a=0.8 \\
\text { Recovery (\%) }\end{array}$} & \multicolumn{2}{|c|}{$\begin{array}{l}a=0.9 \\
\text { Recovery }(\%)\end{array}$} \\
\hline & & & OXY & OXF & OXY & OXF & OXY & OXF & OXY & \\
\hline 1 & 20 & 2 & $\begin{array}{c}288.6 \\
99.21\end{array}$ & $\begin{array}{l}296.3 \\
94.43\end{array}$ & $\begin{array}{c}286.1 \\
98.93\end{array}$ & $\begin{array}{c}292.4 \\
94.37\end{array}$ & $\begin{array}{c}283.4 \\
98.80\end{array}$ & $\begin{array}{c}288.2 \\
99.75\end{array}$ & $\begin{array}{l}281.3 \\
99.71\end{array}$ & $\begin{array}{l}284 \\
99.32\end{array}$ \\
\hline 2 & 20 & 14 & 99.10 & 100.90 & 100.35 & 100.53 & 99.57 & 102.02 & 100.80 & 101.74 \\
\hline 3 & 20 & 26 & 104.47 & 101.78 & 104.89 & 101.29 & 101.86 & 101.88 & 94.94 & 102.31 \\
\hline 4 & 4 & 10 & 94.52 & 101.61 & 95.86 & 101.40 & 96.51 & 101.73 & 100.00 & 101.45 \\
\hline 5 & 16 & 10 & 99.03 & 97.00 & 99.30 & 96.47 & 98.84 & 98.00 & 100.99 & 97.43 \\
\hline \multirow[t]{4}{*}{6} & 28 & 10 & 97.21 & 96.65 & 98.10 & 96.25 & 98.53 & 99.52 & 99.52 & 99.38 \\
\hline & & mean & 98.9 & 98.7 & 99.6 & 98.4 & 99.0 & 100.5 & 99.3 & 100.3 \\
\hline & & SD & 3.26 & 3.10 & 3.01 & 3.05 & 1.73 & 1.65 & 2.23 & 1.87 \\
\hline & & RSD & 3.30 & 3.14 & 3.02 & 3.10 & 1.75 & 1.64 & 2.24 & 1.86 \\
\hline
\end{tabular}

$S D$ standard deviation, $R S D$ relative standard deviation

This approach is denoted as MEXH-CWT-MOFrFT. Figures $2 b, 3 b$, $4 \mathrm{~b}$ and $5 \mathrm{~b}$ show the MOFrFT-CWT spec- tra of OXF and OXY. The similar procedure is used to obtain the MOFrFT-CWT spectra of the artificial and commercial veterinary samples of the related compounds.

The calibration graphs were obtained by the least square calculation based on the relationship between the concentration and the MOFrFT-CWT signals. The linear regression analysis and its statistical results obtained by the MOFrFT- CWT at different parameters are summarized in Table 1 . The results indicate that the proposed signal processing tools pro- vides good correlation coefficients.

The applicability and validity of the proposed MOFrFT- CWT approaches was performed by analyzing the artificial
OXF-OXY mixtures. The recovery and the relative standard deviations are presented in Table 2. As it can be seen from this table, the MOFrFT-CWT approaches at $a=0.8$ lead to the lowest standard deviation with relative standard deviation. This indicates that the method under $a=0.8$ provides a good selectivity for the analysis of OXF and OXY in samples. Limit of detection (LOD, signal to noise $=3$ ) and limit of quantization (LOQ, signal to noise $=10$ ) were calculated by using the standard deviation of the intercepts of linear regression functions (see Table 1).

In this paper, the proposed MOFrFT-CWT signal process- ing methods were successful applied to the commercial vet- erinary samples. Table 3 depicts the quantitative estimation results of the related substances in samples. These results 
Table 3 Quantitative estimation results of OXY and OXF in the commercial veterinary samples by the new proposed

\begin{tabular}{|c|c|c|c|c|c|c|c|c|}
\hline \multirow[t]{2}{*}{ No. } & \multicolumn{2}{|l|}{$a=0.6$} & \multicolumn{2}{|c|}{$a=0.7$} & \multicolumn{2}{|c|}{$a=0.8$} & \multicolumn{2}{|c|}{$a=0.9$} \\
\hline & OXF & OXY & OXF & OXY & OXF & OXY & OXF & OXY \\
\hline & 288.6 & 296.3 & 286.1 & 292.4 & 283.4 & 288.2 & 281.3 & 284 \\
\hline 1 & 591.2 & 304.4 & 596.8 & 303.0 & 594.8 & 302.7 & 595.8 & 311.7 \\
\hline 2 & 574.7 & 296.7 & 581.6 & 295.1 & 585.4 & 301.3 & 581.9 & 302.7 \\
\hline 3 & 575.5 & 299.9 & 581.2 & 298.0 & 579.6 & 304.3 & 580.9 & 306.2 \\
\hline 4 & 594.7 & 305.5 & 597.4 & 303.6 & 594.2 & 307.9 & 594.3 & 312.0 \\
\hline 5 & 577.4 & 296.5 & 579.6 & 294.8 & 577.2 & 301.1 & 578.5 & 303.4 \\
\hline 6 & 553.2 & 296.3 & 558.9 & 295.1 & 569.4 & 301.2 & 563.7 & 301.3 \\
\hline mean & 577.8 & 299.9 & 582.6 & 298.2 & 583.5 & 303.1 & 582.5 & 306.2 \\
\hline SD & 14.75 & 4.14 & 14.1 & 4.1 & 9.99 & 2.68 & 11.73 & 4.65 \\
\hline RSD & 2.55 & 1.38 & 2.4 & 1.4 & 1.71 & 0.88 & 2.01 & 1.52 \\
\hline
\end{tabular}

show that the proposed signal processing tools based on the combined use of the MOFrFT, and CWT can be applied to the quality control and routine analysis of OXF and OXY in commercial samples.

\section{Conclusions}

The challenges of the spectral multicomponent analysis are to find the optimal denoising, smoothing and resolved peak resolution techniques to obtain better determination results of analyses in complex mixture systems. As a result, the FrFT approach combined with CWT is one opportunity to over- come the above mentioned challenges. In this study, FrFT approaches for differentscale parameterfollowed by MEXH-CWT provided the successful quantitative analysis results because of the strong overlapping spectral bands of OXF and OXY in the same spectral region. This combined application of MEXH-CWT-MOFrFT gave the lateral analytical evalua- tion of the content of the commercial veterinary samples. We conclude that the new combined proposed signal processing approaches are accurate, precise, rapid, economic and pow- erful tools for the quality control and quantitative evaluation of the veterinary samples of the related drugs.

Acknowledgments This work was done within the Chemometric Laboratory of Faculty of Pharmacy, and it was supported by the sci- entific research project No. 10A3336001 of Ankara University, Turkey.

\section{References}

1. Gunes, V., Inci, A., Uyanik, F., Yildirim, A., Altug, N., Eren, M., Onmaz, A.C., Gelfert, C.C.: The effect of oxfendazole plus oxy- clozanide paste and tablet formulations on parasite burden and metabolic status of sheep. J. Animal Vet. Adv. 5(7), 589594 (2008)

2. Dinç, E., Baleanu, D., Tokar, F.: Simple mathematical resolution for binary mixture of oxfendazole and oxyclozanide in bolus by bivariate and multivariate calibrations based on the linear regression functions. Revue Roumaine de Chimie 4(53), 303-307 (2008)

3. Dinç, E., Baleanu, D.: Continuous wavelet transform applied to the overlapping absorption signals and their ratio signals for the quantitative resolution of mixture of oxfendazole and oxyclozanide in bolus. J. Food Drug Anal. 2(15), 109-117 (2007)

4. Dinç, E., Kanbur, M.: Spectrophotometric multicomponent res- olution of a veterinary formulation containing oxfendazole and oxyclozanide by multivariate calibrationprediction techniques. J. Pharm. Biomed. Anal. 3-4(28), 779788 (2002)

5. Khan, A.R., Akhtar, M.J., Mahmood, R., Ahmed, S.M., Malook, S., Iqbal, M.: LC assay method for oxfendazole and oxyclozanide in pharmaceutical preparation. J. Pharm. Biomed. Anal. 1(22), 111-114 (2000)

6. Dinç, E., Onur, F.: Comparative study of the ratio spectra derivative spectrophotometry, derivative spectrophotometry and Vierordt's method applied to the analysis of oxfendazole and oxyclozanide in a veterinary formulation. Analysis 3(25), 55-59 (1997)

7. Condom, E.U.: Immersion of the Fourier transform in a continuous group of functional transformations. Proc. Natl. Acad. Sci. 3(23), 158-164 (1937)

8. Ozaktas, H.M., Ankan, O., Kutay, M.A., Bozdag־i, G.: Digital com- putation of the fractional Fourier transform. IEEE Trans. Signal Process. 44(9), 2141-2150 (1996)

9. Tao, R., Deng, B., Zhang, W.-Q., Wang, Y.: Sampling and sampling rate conversion of band limited signals in the fractional Fourier transform domain. IEEE Trans. Signal Process. 56(1), 158-171 (2008)

10. Ozaktas, H.M., Zalesvsky, Z., Kutay, M.A.: The Fractional Fourier Transform. Wiley, Chichester (2001)

11. Narayanan, V.A., Prabhu, K.M.M.: The fractional Fourier trans- form: theory, implementation and error analysis. 
Microprocess. Microsyst. 27(10), 511-521 (2003)

12. Bultheel, A., Martínez Sulbaran, H.: Computation of the fractional Fourier transform. Appl. Comput. Harmon. Anal. 16(3), 182-202 (2004) 
13. Campos, R.G., Rico-Melgoza, J., Chavez, E.: XFT: extending the digital application of the Fourier transform. http://www.citebase.

org/abstract?id=oai:arXiv.org:0911.0952

14. Saxena, R., Singh, K.: Fractional Fourier transform: a novel tool for signal processing. J. Indian Inst. Sci. 85, 11-26(2005)
15. Pei, S.-C., Ding, J.-J.: Relations between Gabor transforms and fractional Fourier transforms and their applications for signal processing. IEEE Trans. Signal Process. 55(10), 48394850(2007) 